\title{
A Methodology of Integrating Marketing with Engineering for Defining Design Specifications of New Products
}

\begin{abstract}
In the fuzzy front end stage of new product development, it is quite common that marketing personnel and product engineers have different goals and concerns. Marketing personnel stress on market surveys, identifying market opportunities and new product positioning, choosing the right price tier, understanding customer preference using data etc. While for product engineers, they concern about defining design specifications of new products in order to satisfy customer needs during the fuzzy front end stage. The two sets of concerns are always addressed in isolation from one another. This isolation typically would not result in optimal design decisions as two sets of concerns are always interrelated. Therefore, it is important to integrate the concerns of marketing personnel with those of engineers in defining design specifications which is mainly about the generation of design attributes and the determination of value setting of them of a new product. Perceptual mapping is a very common technique used by marketing personnel to understand market positions of competitive products and help define new product opportunities. Although quite a few research works have been attempted to integrate marketing with engineering concerns for new product design, perceptual mapping was not considered in defining design specifications of a new product in the previous related studies. Failure to do that would lead to a new product with its position identified through the perceptual mapping may not be feasible for product development in terms of technical considerations. In this paper, a methodology of integrating marketing with engineering for defining design specifications of new products is described which mainly involves generation of perceptual maps, generation of fuzzy regression models for relating customer requirements and design attributes, formulation of an optimization model and solving the model based on genetic algorithms. In the proposed methodology, factor analysis is involved to construct a perceptual map by which a region of new product opportunities can be identified. An optimization model for determining target value setting of design attributes of a new product is developed in which the Kano model is introduced to formulate its objective function. The region identified from the perceptual map is then transformed to a mathematical constraint of the optimization model. A genetic algorithm is employed to solve the optimization problem and optimal target value setting of
\end{abstract}


design attributes of the new product can be determined. A case study of defining design specifications of a new packing machine was used to illustrate the proposed methodology.

Keywords: New product design; Design specifications; Design attributes; Product positioning.

\section{Nomenclature}

$\mathrm{CR}_{i}$ : the $i$ th customer requirement, $i=1,2, \cdots, m$;

$\mathrm{DA}_{j}$ : the $j$ th design attributes, $j=1,2, \cdots, n$;

$\mathrm{Comp}_{h}$ : the $q$ th competitor, $h=1,2, \cdots, q$;

$y_{i}$ : the satisfied degree of performance of $\mathrm{CR}_{i}, \quad i=1,2, \cdots, m$;

$w_{i}$ : the relative importance of the $\mathrm{CR}_{i}$, and $\sum_{i=1}^{m} w_{i}=1$;

$x_{j}$ : the fulfillment degree of $\mathrm{DA}_{j}, \quad 0 \leq x_{j} \leq 1, j=1,2, \cdots, n$;

$f_{k}$ : the $k$ th underlying factor, $k=1,2, \cdots, Z$

$r_{i}$ : the functional relationship between $\mathrm{CR}_{i}$ and DAs, $i=1,2, \cdots, m$;

$g_{j}$ : the functional relationship between $\mathrm{DA}_{j}$ and other DAs, $j=1,2, \cdots, n$;

$q_{k}$ : the functional relationship between $k$ th underlying factor and CRs, $k=1,2, \cdots, \mathrm{Z}$

$C:$ the total cost of product development;

$C_{\mathrm{F}}$ : the fix parts of $C$;

$C_{\mathrm{V}}:$ the variable part of $C$;

$C_{j}$ : the cost for achieving $x_{j}, \quad j=1,2, \cdots, n$;

$c_{j}$ : the cost per unit of $x_{j}, j=1,2, \cdots, n$;

$B$ : the budget of product development. 


\section{Introduction}

A successful product design requires, among others, an efficient and effective integration of engineering and marketing. However, the entire product development process typically is broken down into a number of stages that are addressed separately in product design optimization. For example, marketing personnel may concern market opportunities and positioning, choosing the right price and understanding 'consumer needs' using data while design engineers emphasize on delivering an optimal, feasible design given a set of desired performance targets, features and costs. In short, the two communities have different languages and notions of drivers of success, optimization variables and the nature of constraints. Formal models / methodologies to combine them are necessary.

Some recent research attempting to link up marketing and engineering concerns for new product development has been conducted. Michalek et al (2005) adopted an analytical target cascading (ATC) method to formalize the process of coordinating marketing and engineering design problems in order to yield a joint optimal solution. Results indicated that the most profitable achievable product can fall short of predictions based on marketing alone but well ahead of what engineering may produce based on original marketing target specifications. Later, Michalek et al (2006) proposed another ATC method that coordinates marketing and design perspectives by incorporating quantitative models of manufacturing investment and production allocation. The effectiveness of the resulting method has been demonstrated on allowing a quantitative assessment of tradeoffs among product functionality, market performance and manufacturing costs to achieve product line solutions. Luo et al (2005) proposed a multi-objective genetic algorithm for optimizing both robustness in engineering performances and consumer preferences so as to evolve new design portfolios. Besharati et al (2006) proposed a multi-objective genetic algorithm that interrelates both engineering design domain and customer preferences in marketing domain. The overall goal of the method is to obtain design alternatives that seeks multi-objective product design alternative in robustness in engineering design and optimum in customer preferences. Kang et al (2007) proposed a methodology of integration of marketing and R\&D for new product development which involves the methods of quality function deployment (QFD), multivariate statistical analysis, conjoint analysis and / or the Taguchi method. All the above studies only address the integration issue for a single product design. Recently, Kumar et al (2008) proposed a methodology of integrating marketing with engineering concerns for product family design. The methodology involves market segmentation grids and the nested logit demand model. In addition, a cost-based approach was adopted in their work to develop a cost model. However, 
in product family planning, structures of product variants sometimes are not well defined such that product cost estimation based on a cost-based approach may not be feasible. Jiao and Zhang (2007) employed conjoint analysis and MNL choice models to formulate the product portfolio planning problem, which links up the marketing concerns with the determination of product specifications and manufacturing cost for product portfolio planning. A heuristic genetic algorithm was proposed to solve the product portfolio planning problem that leverages both the customer and engineering concerns.

Perceptual mapping is a very common technique used by marketing personnel to understand market positions of various competitive products and help define new product opportunities based on perceptual maps. However, the previous studies did not consider perceptual mapping in defining design specifications of a new product. Failure to do that would lead to a new product with its position identified through perceptual mapping may not be feasible for product development in terms of technical aspect. In this paper, a methodology of integrating marketing with engineering for defining design specifications of new products is proposed to address the deficiency. The methodology mainly involves the generation of perceptual maps, generation of fuzzy regression models for relating customer requirements (CRs) and design attributes (DAs) as well as a design attribute and other design attributes, formulation of an optimization model and solving the model based on genetic algorithms.

The rest of the paper is organized as follows. In the next section, a methodology of integrating marketing and engineering for defining design specifications of new products is described in details. In section 3, a case study of defining design specifications of a new packing machine based on the proposed methodology is provided to illustrate and evaluate the effectiveness of the proposed methodology. Finally, conclusions and future work are presented in section 4 .

\section{A methodology of integrating marketing and engineering for defining design specifications}

Figure 1 shows the proposed methodology of integrating marketing and engineering for defining design specifications of new products. A design specification normally consists of a design attribute and its value. Defining design specifications is an important process of a new product development project as the specifications would largely affect the downstream product development activities. The proposed methodology contains five main steps which 
are construction of a house of quality (HOQ), generation of perceptual maps and identification of region of new product opportunities, generation of fuzzy regression models for relating customer requirements and design attributes as well as a design attribute and other design attributes, formulation of an optimization model and solving the model based on genetic algorithms. Since construction of a HOQ has been described in a number of publications, it will not be described here. Details of other steps of the methodology are described as follows.

[insert Figure 1 about here]

\subsection{Construction of perceptual maps and identification of regions of new product opportunities}

Once a HOQ is developed, a perceptual map can be generated. A perceptual map is a graphical representation in which competing alternatives are plotted in an Euclidean space. With the aids of the maps, marketing personnel could understand marketing positions of various competitive products, identify possible new product opportunities, determine position of a new product, and clusters of competitive products as well as identify possible major competitive products. It can be made in three ways by: determinant perceptual mapping, attribute rating (AR) perceptual mapping, and overall similarities (OS) perceptual mapping (Crawford and Benedetto 2003). AR perceptual mapping has been adopted in this research which makes use of customer attribute ratings to obtain data from users. It always involves statistical techniques such as factor analysis and multiple discriminant analysis. In this research, factor analysis was adopted to construct the map based on competition benchmarking data of the HOQ. Factor analysis (Johnson and Wichern, 1992) is a statistical technique which is commonly used to uncover the latent structure (dimensions) of a set of variables. It reduces attribute space from a larger number of variables to a smaller number of factors and as such is a "non-dependent" procedure. To apply factor analysis on generating perceptual maps, first, the correlations among all customer requirements (CRs) are calculated using benchmarking data of the HOQ. Then, underlying factors are extracted from the correlation matrix. Factor loadings are then calculated which are the correlation coefficients between the variables and factors. The next step is to derive factor scores. Factor scores are the scores of each case on each factor. After that, underlying factors are rotated. The purpose of the rotation is to make the output more understandable and is usually necessary to facilitate the interpretation of factors. The underlying factors can then be interpreted. Through the 
application of the above procedures, the relationships between the underlying factors and CRs can be expressed as follows.

$$
f_{k}=\sum_{i=1}^{m} d_{k i} y_{i}, \quad k=1, \cdots, z
$$

where $d_{k m}$ is the value of factor score between the k-th factor and the i-th CR.

The two factors with the largest values of factor loading are selected as the $\mathrm{x}$ axis and $y$ axis of the perceptual map respectively. Positions of the competitive products which are used for the benchmark in the HOQ on the map can be determined using (1). From the map, regions of new product opportunities may be identified by marketing personnel. After evaluating the regions, a region of new product opportunities which is believed to have the highest potential for developing a successful new product would be identified. The region is then converted into a mathematical model as expressed in (2) which will be one of the constraints of an optimization model to be formulated later.

$$
\phi_{\lambda}=p_{\lambda}(\boldsymbol{f}) \leq 0, \lambda=1, \cdots, \eta
$$

where $\phi_{\lambda}$ represents the $\lambda$-th region of new product opportunities of a new product, and $p_{\lambda}(\boldsymbol{f})$ is a mathematical function for describing $\phi_{\lambda}$.

\subsection{Generation of models for relating CRs and DAs as well as a DA and other DAs}

This step is about the development of the models for relating CRs and DAs as well as a DA and other DAs based on a HOQ. Various approaches have been attempted in the modeling including statistical regression (Dawson and Askin 1999) and fuzzy regression (Fung et al 2006, Chen and Chen 2006). As modeling the relationships based on fuzzy regression can yield explicit models using a small number of data sets, it was adopted in this research to model the relationships. In addition, fuzziness of the relationships between CRs and DAs as well as a DA and other DAs can be addressed by fuzzy regression properly. Details of the modeling using fuzzy regression can be found in the authors' publications (Chen et al 2004, Kwong and Bai 2005). 


\subsection{Formulation of an Optimization Model}

Once functional relationships $r_{i}(x), i=1, \ldots, m$, have been modeled, a function for deriving overall customer satisfaction (OCS) can be constructed by aggregating various dimensions of customer satisfaction $y_{i}, i=1, \ldots, m$, and their importance weights $w_{i}, i=1, \ldots, m$. This can be regarded as the multiple attribute synthesis at the system level. In most previous studies, the linear weighted sum method (WSM) was used to derive the overall customer satisfaction (OCS), which can be expressed as follows.

$$
\mathrm{OCS}=\sum_{i=1}^{m} w_{i} y_{i}
$$

However, it is difficult to justify in general that all customer requirements would contribute to customer satisfaction at the same level. According to the Kano model as shown in the figure 2, usually there are three major types of customer satisfaction. The first, depicted by the diagonal line, represents explicit requirements, which are directly related to customer satisfaction. The second type represents innovations, as shown by the curved line in the upper left corner of the figure. If the associated requirements are fufilled, customer satisfaction will increase dramatically. The third one represents "must-have" requirements, as shown by the lower right curve of the figure. When these customer requirements do not exist, the customers will be very dissatisfied; but if they do exist, they are taken for granted, and customer satisfaction remains relatively stable.

\section{[Insert Figure 2 about here]}

To consider different kinds of customer requirements as depicted in the Kano model in real-world problems, a new objective function of deriving OCS based on a generalized weighted mean method (Kano, 1984) has been constructed and is presented as follows:-

$$
\text { OSC }=\left(\sum_{i=1}^{k} \varpi_{i} y_{i}^{s}\right)^{s}+\left(\sum_{i=k+1}^{l} \varpi_{i} y_{i}\right)+\left(\sum_{i=l+1}^{m} \varpi_{i} y_{i}^{\frac{1}{t}}\right)^{t}
$$

$y_{i}$ with $i=1, \ldots, k$ denotes the customer satisfaction value of "Attractive" type customer requirements. $y_{i}$ with $i=k+1, \ldots, l$ denotes the customer satisfaction value of "explicit" type customer requirements. $y_{i}$ with $i=l+1, \ldots, m$ denotes the customer satisfaction value of "must-be" type customer requirements. The value of $s$ and $t$ are dependent on the nature of the new product design.

An optimization model is formulated to determine a set of level of attainment of DAs, $x_{1}, x_{2}, \cdots x_{n}$, for a new product in order to maximize the OCS subject to the product positioning constraints and other constraints. If the $\lambda$ th region of new product opportunities 
is selected from the perceptual product map, the optimization model of integrating marketing with engineering for determining value setting of design attributes of new products can be formulated as the following Kano model:

$$
\text { OCS }=\left(\sum_{i=1}^{k} \varpi_{i} y_{i}^{s}\right)^{s}+\left(\sum_{i=k+1}^{l} \varpi_{i} y_{i}\right)+\left(\sum_{i=l+1}^{m} \varpi_{i} y_{i}^{\frac{1}{t}}\right)^{t}
$$

Subject to

$$
\begin{aligned}
& y_{i}=f_{i}(\boldsymbol{x}), \quad i=1,2, \cdots, m \\
& x_{j}=g_{j}\left(\boldsymbol{x}^{j}\right), \quad j=1,2, \cdots, n \\
& \phi_{\lambda}=p_{\lambda}(\boldsymbol{f}) \leq 0, \quad \lambda=1, \cdots, \eta \\
& y_{i}^{\min } \leq y_{i} \leq y_{i}^{\max }, \quad i=1,2, \cdots, m \\
& 0 \leq x_{j} \leq 1, j=1,2, \cdots, n
\end{aligned}
$$

where (6) refers to the models of the functional relationships between $\mathrm{CR}_{i}$ and DAs. (7) refers to the models of the functional relationships among DAs. (8) refers to the constraint of the position of the new product; (9) and (10) refers to the ranges of customer satisfaction values and levels of attainment of $\mathrm{DA}_{j}$ respectively.

\subsection{Solving the optimization model}

Since the model (5-10) is a non-linear type, genetic algorithms (GAs) are found suitable to solve this type of optimization problem. When the objective functions to be optimized in optimization problems are multi-modal or the search spaces are particularly irregular, algorithms need to be highly robust in order to avoid getting stuck at a local optimal solution. Genetic algorithms (Reeves 1997) have become increasingly popular for searching good solutions in many hard optimization problems. It can be found from many literatures (Chatterjee et al 1995, Chu and Beasley 1997, Lin et al 1995) that they can find good or reasonable solutions.

\section{$3 \quad$ An illustrative Example}

A case study of defining design specifications of a new packing machine based on the proposed methodology was implemented using Matlab programming software. Figure 3 shows a HOQ for the packing machine design (Chen et al, 2004). In the machine design, four 
major CRs are identified based on a customer survey which are "quality of packing" $\left(\mathrm{CR}_{1}\right)$, "efficiency of packing" $\left(\mathrm{CR}_{2}\right)$, "packing noise" $\left(\mathrm{CR}_{3}\right)$ and "rigidity of the machine" $\left(\mathrm{CR}_{4}\right)$. Seven DAs are identified, which are "precision of the molding of the clip" (DA $)$, "precision of the packing" $\left(\mathrm{DA}_{2}\right)$, "control force of the packing" $\left(\mathrm{DA}_{3}\right)$, "efficiency of the packing" $\left(\mathrm{DA}_{4}\right)$, "hardiness of the pressing hammer" $\left(\mathrm{DA}_{5}\right)$, "noise of the cam power transmission" $\left(\mathrm{DA}_{6}\right)$ and "height of the machine bed" $\left(\mathrm{DA}_{7}\right)$. Five major competitive products are identified which are denoted as Comp1, Comp2, Comp3, Comp4 and Comp5 respectively. With the use of the factor analysis as described in Section 2, two factors with the largest values of factor loading were determined, which are denoted as $f_{1}$ and $f_{2}$ respectively. Results of the factor analysis are shown in Table 1 and Table 2. Table 1 shows the rotated factor loadings and communalities of each dimension of customer satisfaction while Table 2 shows the factor score coefficients of $f_{1}$ and $f_{2}$.

[Insert Figure 3, Table 1 and Table 2 about here]

From Table 1 , it can be observed that $f_{1}$ is dominated by $\mathrm{CR}_{1}$ and $\mathrm{CR}_{2}$ while $f_{2}$ is dominated by $\mathrm{CR}_{3}$ and $\mathrm{CR}_{4}$. From Table 2, the relationships between the two underlying factors and the four CRs can be written as follows:

$$
\begin{aligned}
& f_{1}=0.464 \mathrm{y}_{1}+0.449 \mathrm{y}_{2}-0.217 \mathrm{y}_{3}+0.166 \mathrm{y}_{4} \\
& f_{2}=-0.030 \mathrm{y}_{1}-0.100 \mathrm{y}_{2}-0.508 \mathrm{y}_{3}-0.695 \mathrm{y}_{4}
\end{aligned}
$$

where $y_{i}(i=1$ to 4$)$ is the degree of satisfaction of $C R_{i}$.

With the use of the above two models, a perceptual map was constructed as shown in Figure 4. There are three regions of possible new product opportunities. Among the three, region 1 was identified as the highest potential one in which a successful new product would be developed. The inputted regions are commonly in forms of circles, ellipses and rectangles. In the paper, it is assumed that the form of region 1 is a circle. The region is converted to a mathematical constraint for new product positioning as described below:

$$
p(\boldsymbol{f})=\left(f_{1}-1.3\right)^{2}+\left(f_{2}-1\right)^{2} \leq 0.5^{2}
$$

Any point within or on the boundary of the region can be expressed using the following parametric equation.

$$
p(\boldsymbol{f})=\left\{\begin{array}{l}
f_{1} \leq 1.3+0.5 \cos \theta \\
f_{2} \leq 1+0.5 \sin \theta
\end{array}, \theta \in[-2 \pi, 2 \pi]\right.
$$


Modeling the functional relationships between the four CRs and the seven DAs as well as those among the seven DAs based on fuzzy regression with symmetric triangular functions, were implemented using Matlab and the function relationship models developed were shown as follows.

$$
\begin{aligned}
& y_{1}=5.98 x_{1}-0.33 x_{2}-1.37 x_{3}+0.88 \\
& y_{2}=2.45 x_{3}+0.96 x_{4}+1.25 x_{5}+0.54 \\
& y_{3}=4.20 x_{6}+1.00 \\
& y_{4}=4.00 x_{7}+1.25 \\
& x_{2}=0.97 x_{4}+0.01 \\
& x_{4}=0.87 x_{2}-0.30 x_{6}+0.22 \\
& x_{6}=-0.87 x_{4}+0.89
\end{aligned}
$$

with $x_{i}(i=1$ to 7$)$ be the level of attainment of $D A_{i}$

and $x_{i}=\frac{l_{i}^{\max }-x_{i}}{l_{i}^{\max }-l_{i}^{\min }}$

where $l_{i}^{\max }$ and $l_{i}^{\min }$ are determined by consideration of competition requirement and technology feasibility [Zhou 1998].

In the HOQ of the packing machines, there are four customer requirements; $\mathrm{CR}_{1}, \mathrm{CR}_{2}$, $\mathrm{CR}_{3}$, and $\mathrm{CR}_{4}$. The design team categorized the $\mathrm{CR}_{4}$ and $\mathrm{CR}_{2}$ as "must be" requirements, $\mathrm{CR}_{1}$ as an explicit requirements and the $\mathrm{CR}_{3}$ as an "attractive" requirements. With the above categorization, the objective function was constructed to derive the overall customer satisfaction, osc, as follows:

$$
\text { OSC }=\left(w_{3} y_{3}^{s}\right)^{1 / s}+w_{1} y_{1}+\left(w_{2} y_{2}^{1 / t}+w_{4} y_{4}^{1 / t}\right)^{t}
$$

Based on the team's knowledge of the packing machine design, it was estimated that both the value of $s$ and $t$ are 2 . With reference to the optimization model (5-10), an optimization model for determining target value setting of design attributes of the new packing machine can be formulated as follows.

$$
\text { OSC }=\left(w_{3} y_{3}^{2}\right)^{1 / 2}+w_{1} y_{1}+\left(w_{2} y_{2}^{1 / 2}+w_{4} y_{4}^{1 / 2}\right)^{2}
$$

Subject to: 


$$
\begin{aligned}
& y_{1}=5.98 x_{1}-0.33 x_{2}-1.37 x_{3}+0.88 \\
& y_{2}=2.45 x_{3}+0.96 x_{4}+1.25 x_{5}+0.54 \\
& y_{3}=4.20 x_{6}+1.00 \\
& y_{4}=4.00 x_{7}+1.25 \\
& x_{2}=0.97 x_{4}+0.01 \\
& x_{4}=0.87 x_{2}-0.30 x_{6}+0.22 \\
& x_{6}=-0.87 x_{4}+0.89 \\
& \left(f_{1}-1.3\right)^{2}+\left(f_{2}-1\right)^{2} \leq 0.5^{2} \\
& f_{1}=0.464 y_{1}+0.449 y_{2}-0.217 y_{3}+0.166 y_{4} \\
& f_{2}=-0.030 y_{1}-0.100 y_{2}-0.508 y_{3}-0.695 y_{4} \\
& 1 \leq y_{i} \leq 5, \quad i=1, \cdots, 4 \\
& 0 \leq x_{j} \leq 1, \quad j=1, \cdots, 7
\end{aligned}
$$

GA was then applied on solving the above model for determining target value setting of DAs. A parameters setting of GA is summarized in Table 3. Figure 5 shows the optimal position, $\diamond$, of the new product based on the proposed methodology. In the figure, the '*' is referred to the position of the new product which was identified using Chen et al's method (2004). It can be seen that the position of the ' ${ }^{*}$ ' is beyond the region 1 which was identified by marketing personnel. The optimal attainment levels of the design attributes obtained by the GA optimization are summarized in Table 4. Hence, the target value setting of the design attributes can be determined as shown in Table 5. The optimal results of the degree of satisfaction for customer requirements are shown in Table 6. Figure 6 shows the convergence plot of the GA. From the figure, it can be found that the GA progressed sharply in the early iterations and saturated in the late iterations. Finally, it converged before the pre-defined number of iterations, which took 425 seconds. The computational time is considered to be reasonable.

[Insert Figure 5 and Figure 6 about here]

[Insert Tables 3, 4, 5 and 6 about here] 


\section{Conclusions and Future Work}

Perceptual mapping is a very common technique used by marketing personnel to understand market positions of their competitive products as well as defining regions of new product opportunitiew. Although quite a few previous studies have attempted to integrate marketing concerns with engineering concerns for product design, none of them has considered perceptual mapping in defining design specifications of new products. In this paper, a methodology for integrating marketing with engineering for defining design specifications of new products is proposed. In the proposed methodology, a factor analysis is used to generate perceptual maps by which a region of new product opportunities can be identified. The region is then converted into a mathematical constraint of an optimization model for determining a target value setting of design attributes. A Kano model was employed to develop an objective function of the optimization model. By solving the model using a genetic algorithm, a target value setting of design attributes of a new product can be obtained by maximizing overall customer satisfaction. A case study for defining design specifications of a new packing machine based on the proposed methodology was carried out to illustrate the proposed methodology. Further work of this project would involve the considerations of price positioning and value of a new product in determining a value setting of design attributes.

\section{Acknowledgement}

This research is financially supported by the Research Grant Council of Hong Kong, China (Project no. PolyU 5184/07E)

\section{References}

Besharati B., Luo L., Azarm S. and Kannan P.K. (2006) Multi-objective single product robust optimization: an integrated design and marketing approach. Transactions of the ASME 128(4), 884-892.

Chatterjee S., Carrera C. and Lynch L.A. (1995) Genetic algorithms and travelling salesman problems. European Journal of Operational Research, 93, 490-510.

Chen, Y. and Chen, L. (2006) A non-linear possibilistic regression approach to model functional relationships in product planning. International Journal of Advanced Manufacturing Technology, 28(11-12), 1175-1181.

Chen, Y.Z., Tang, J.F., Fung, R.Y.K. and Ren, .ZH. (2004) Fuzzy regression - based 
mathematical programming for quality function deployment. International Journal of Production Research 42(5), 1009-1027.

Chu P.C. and Beasley J.E. (1997) A genetic algorithm for the generalised assignment problem. Computers Operations Research, 24(1), 17-23.

Crawford, M. and Benedetto A. (2003) New products Management, McGraw Hill.

Dawson, D. and Askin R.G. (1999) Optimal new product design using quality function deployment with empirical value functions, Quality and Reliability Engineering International, 15, 17-32.

Fung, R.Y.K., Chen, Y., and Tang, J. (2006) Estimating function relationships for product planning under uncertainties. Fuzzy Sets and Systems, 157, 98-122.

Jiao, J. and Zhang, Y. (2007) Product portfolio planning with customer-engineering interaction. IIE Transactions, 37(9), 801-814.

Johnson, R.A. and Wichern, D.W. (1992) Applied multivariate statistical analysis, Prentice Hall, Englewood Cliffs, New Jersery.

Kang, N., Kim, J. and Park, Y. (2007) Integration of marketing domain and R\&D domain in NPD design process. Industrial Management and Data Systems, 107(6), 780-801.

Kano, N. (1984) Attractive quality and must-be quality. The Journal of the Japanese Society for Quality Control, 14(2), 39-48.

Kumar, D., Chen, W. and Simpson, T.W. (2008) A market-driven approach to product family design. International Journal of Production Research (in print)

Kwong, C.K., and Bai, H., (2005) Fuzzy Regression Approach to Process Modeling and Optimization of Epoxy Dispensing. International Journal of Production Research, 43(12), 2359-2375.

Lin C.K.Y., Haley K.B. and Sparks C. (1995) A comparative study of both standard and adaptive versions of threshold accepting and simulated annealing algorithms in three scheduling problems. European Journal of Operational Research, 83, 330-346.

Luo L., Kannan P.K., Besharati B. and Azarm S. (2005) Design of robust new products under variability: marketing meets design. Journal of Product Innovation Management, 22, 177-192.

Michalek J.J., Feinberg F.M. and Papalambros P.Y. (2005) Linking marketing and engineering product design decisions via analytical target cascading. Journal of Product Innovation Management, 22, 42-62.

Michalek J.J., Ceryan O., Papalambros P.Y. and Koren Y. (2006) Balancing marketing and manufacturing objectives in product line design. Transactions of the ASME, 128(6), 
1196-1204.

Reeves C. (1997), Genetic algorithms for operations researcher. INFORMS Journal on Computing, 9(3), 231-250.

Zhou, M. (1998) Fuzzy logic and optimization models for implementing QFD. Computers and Industrial Engineering, 35,237-240. 